\title{
Numerical experiments and compartment fires
}

\author{
Nils Johansson
}

\begin{abstract}
Fires are complex and it is hard to derive relationships from theory in fire science. Full-scale and small-scale experiments have been used with great success in order to increase the understanding of fire chemistry and fire dynamics. An alternative or complement to these often expensive and resource demanding traditional experiments are numerical experiments. In this paper, numerical experiments are reviewed as a research method and put into the context of traditional compartment fire experiments. Benefits and challenges with numerical experiments compared to traditional compartment fire experiments are presented and discussed in this paper. Numerical experiments are a promising method in fire science research. However, it is currently not considered satisfying to solely use a numerical experiment to study a certain fire phenomena. Different experimental methods should not be regarded as competitive but as complementary, and a combination of traditional and numerical experimental methods are in many cases appropriate in order to analysis a certain fire phenomena.
\end{abstract}

Keywords: Numerical experiments; Compartment fires; Fire dynamics; FDS; Simulations

\section{Introduction}

Compartment fire dynamics are complex and can only be described analytically with simplified theories due to the random behaviour of fires and flames. Fire experiments are therefore a necessity in order to study and understand the compartment fire dynamics. There is a range of correlations available in the fire science literature, derived with the help of empirical data from experiments, that gives some explanation of different fire phenomena. Fire experiments can be conducted in a variety of ways, e.g. it can be in a limited-scale were only some single variables are studied, in a small-scale and in full-scale.

The cone calorimeter (ISO 2002) is an example of a limited-scale experiment where the reaction to different heat fluxes of materials can be studied. This type of experiment is performed in a controlled environment with high internal validity in order to study relationships between a few variables. Everything except the variables of interest is kept constant and by varying the variables the correlation between them can be studied. Limited-scale experiments can be used to investigate relationships on a fundamental level in fire science. However, results from these experiments are not sufficient in order to

Correspondence: nils.johansson@brand.lth.se

Department of Fire Safety Engineering, Lund University, P.O. Box 118, SE-221 00, Lund, Sweden understand and evaluate fire development and smoke spread in a single and multi-room compartment, i.e. the external validity is low.

A realistic fire environment can be scaled down to an appropriate size with the help of scaling laws. With such a small-scale experimental setup it is possible to conduct the experiments in a controlled environment, i.e. in a fire laboratory. Everything except the variable of interest is kept constant and by varying it, the effect of the variable on the experiment can be analysed. Some compromises are necessary in the small-scale experiments because it is not possible to comply with the scaling laws for all the mechanisms of importance in fire science (Quintiere 2006). To avoid such issues full-scale experiments are necessary.

It is possible to conducted full-scale fire experiments of single apartments or even entire buildings in some indoor fire laboratories in the world, e.g. at SP in Sweden, Underwriters Laboratories and FM Global in USA, but it is harder to control important variables as the experimental setup increases in size. Even well controlled experiments can be difficult to reproduce (Lundin 1999). Also, it might not be possible to conduct the experiment in an indoor laboratory environment due to economical reasons or lack of laboratory space and equipment to handle a large experimental setup. In such a situation the alternative can be to move the experiment outdoors.

\section{Springer}


But, in that case it is even more problematic to control and measure important variables sufficiently accurate. Especially wind speed and wind direction have been seen to have a large influence outdoor full-scale experiments; consequently, it can be very hard to analyse and draw conclusions from such experiments (Johansson et al. 2012; 2014a).

Another option is to use a fire model to study and analyse a certain fire phenomenon. Models includes interpretations and approximations of reality but can be used to help to understand some physical reality (Blurock and Battin-leclerc 2013). Using fire models in an experimental manner can be called numerical experiments and it is considered to be a promising method for research in fire science, but there are both advantages and challenges compared with small- and full-scale experiments.

\section{What is a numerical experiment?}

Both experiments and observations can be used to collect data and test hypotheses. A passive observation involves an investigation of a pre-existing state without attempting to influence it. An example of this in fire science research is when real fire incidents are studied. Collecting data by observing a system and then trying to extract interesting information afterwards is common in research (Andersson 2012), but it is not an experiment.

An experiment is something more than just an observation and collecting data. In e.g. a compartment fire experiment the state of the system (the compartment fire setup) is changed by for instance varying the heat release rate or door opening size and the result of that change in the system is measured and analysed in order to generate information that is relevant to a certain research question (see Figure 1).

The term numerical experiment has been used in other scientific fields for quite some time, e.g. by Bowman et al. (1993), and lately there are also examples of the term being used in fire science (Chow and Zou 2005; Tilley et al. 2012; Johansson and van Hees 2014). However, there is, to the knowledge of the author, no established definition or description of numerical experiment in field of fire science. Therefore, the following definition of numerical experiments is applied in this paper:

\section{A numerical experiment is performed when a numerical model is used in a systematic experimental approach.}

The term experimental approach refers to the description of experiments given in Figure 1, i.e. a systematic manipulation of a system in order to answer a certain research question. This definition makes a clear distinction between numerical experiments and simulations/predictions that are e.g. used for building design. Because the purpose of the latter is normally to demonstrate the performance of a building with regard to some selected fires and not to explain how the studied system works.

Physical experiments, in full- or small-scale, that have traditionally been used to study compartment fires are referred to as traditional experiments in this paper.

\section{Scope of the paper}

The scope of this paper is to review numerical experiments and to explore it as a research method in fire science with a particular focus on compartment fires. This paper is exploratory (Robson 2002; Höst et al. 2006) e.g. insight is sought into how numerical experiments can be used as an experimental method in fire science research. The review is performed with a comparative approach where the advantages and challenges of numerical experiments are compared to traditional experiments, and this is done with several examples of previously performed numerical experiments. Furthermore, factors that might affect the accuracy of numerical experiments are also addressed in this paper, in order to give the reader a possibility to evaluate the applicability of results from a numerical experiment.

The paper is limited to numerical experiments that are used to study compartment fires for research purposes, which means that this work does not deal with standardized testing methods. This does however not exclude the discussion in this paper for being relevant

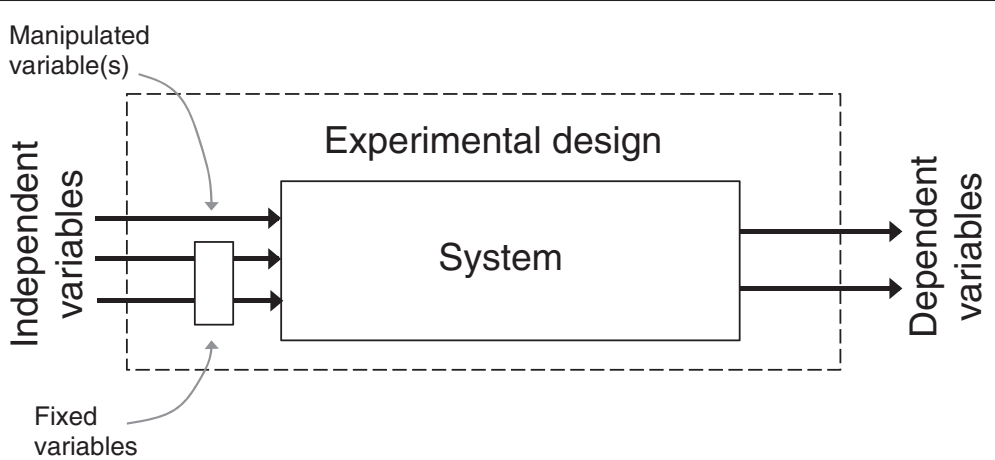

Figure 1 Illustration of experiment, inspired by (Höst et al. (2006). 
when using numerical models as an alternative or complement to fire testing.

It is not the numerical model itself that is of interest in this study but how it can be used as an experimental tool. However, the available computer power and the tools available for fire modelling have developed rapidly during the last decades and this is believed to affect how the models can be used as an experimental tool. This is why the examples of numerical experiments given in this paper are limited to work conducted during the last two decades.

\section{Examples of numerical experiments in fire science} Several studies have been published where numerical methods have been used to study some phenomena in fire science in a way that would fit the presented definition of numerical experiments (see Figure 1). There are several early examples of where numerical investigations have been used as a complement to traditional experiments are e.g. the studies of post-flashover fires by Magnusson and Thelandersson (1970) and the studies of ceiling jets by Alpert (1975). However, this paper is, as stated above, limited to more recent studies and the following examples are used to illustrate how numerical experiments has been used as a research method in fire science.

The National Institute of Standards and Technology (NIST) and Underwriters Laboratories (UL) used the Industrial Fire Simulator (IFS), which essentially corresponded to the first version of the Fire Dynamics Simulator (FDS) (Floyd et al. 2013a), in an extensive research program. The effect of roof vents and draft curtains on the time, number, and location of sprinkler activations and vice versa were studied with both traditional experiments and modelling (McGrattan et al. 1998; McGrattan et al. 2000). The results from the modelling were compared to the experimental results; however, the model was also used to provide information about conditions in the experiment that was not measured. Furthermore, the model was used to study what would happen if some of the test parameters would be varied. In this way, the resources could be optimized because the number of traditional experimental tests did not need to be increased in order to study additional variants of the original experimental setup.

Prasad et al. (2002) performed a large number of simulations with a CFD model, in order to study water-mist suppression in a compartment. The effects of various water mist injection parameters i.e. droplet diameter, mist injection velocity and injection density on the time to fire suppression were studied. The authors state that some of the values for the range of parameters used in the study would not be attainable experimentally because the nozzles needed were not commercially available. But with the CFD model it was possible to study general trends, when the droplet density or injection velocity or droplet diameter was varied systematically. The authors compared modelling results of the ceiling gas temperatures distribution with experimental data in a report issued by the US Naval Research Laboratory (Prasad et al. 2000) and found the results to be favourable. However, the water-mist suppression modelling was not compared to traditional experiments due to lack of experimental data, and that is of course drawback of the study because it can raise questions about the external validity of the of the performed numerical experiment.

$\mathrm{Li}$ and Chow (2003) evaluated different types of ventilation systems in a tunnel with a CFD code. The size of the tunnel was kept constant in the simulations but the fire size, type of ventilation system and its capacity were varied. A comparison of four different tunnel ventilation systems (longitudinal, semi-transverse, transverse and a combination of longitudinal and semi-transverse system) was done based on the simulations, and both advantages and disadvantages of each system were presented. Regarding using numerical experiments in the paper the authors' express that different configurations can be analysed without the need to perform the traditional experimental tests.

Hwang and Edwards (2005) used FDS 2 to study fires in tunnels or more specific the ventilation velocity (critical ventilation velocity) that is just able to prevent the formation of a back-layer. Different heat release rates and tunnels of different sizes were used in the study. The critical ventilation velocity was found to be roughly proportional to the $1 / 5$ power of the heat release rate until a certain critical point. The authors noted that the fireplume velocity had the same dependency on the heat release rate (Quintiere, 1989) and that it can be argued that it is the base velocity of the back-layer velocity. A comparison to data from some traditional experiments was also conducted in the study.

Chow and Zou (2005) used the term numerical experiments when using FDS 3.01 to derive a correlation for doorway flows between two rooms. Chow and Zou first compared results from the computer model with experimental data presented by Nakaya, et al. (1986) and then used FDS to find a value for the constant, $k$, in the well known expression for mass flow through an opening, $\dot{m}=k \cdot A_{0} \sqrt{H_{o}}$ first recognized by Kawagoe (1958). Chow and Zou found that the FDS simulations corresponded well to other prior empirical studies.

Tilley et al. (2011) studied if simulations with FDS 5.2.5 could be used in a numerical experiment by studying the agreement between FDS and experimental data from two different small-scale setups, a tunnel and atria. Tilley et al. describes that the main advantage of numerical experiments is that it is possible to study the effect of a large 
amount of different parameters by varying them in several simulations. Tilley et al. (2011) found that FDS gave good predictions of the smoke layer and that the fire model could be used for a parameter variation study for similar compartment configurations. Tilley et al. (2012) followed up the first study with a study of car parks with FDS in order to create a simple analytical formula for the critical ventilation velocity and backlayering distance in car park fires. Tilley et al. used the data from 350 FDS simulations to develop such a simple analytical expression for a large closed car park with flat ceiling. The convective heat release rate per unit area, the car park height and the car park width were varied in the simulations. Results with the derived formulas were compared to data from a set of full-scale experiments and a reasonable agreement of the smoke backlayering distance was demonstrated (Tilley et al. 2012). The vast number of simulations implies that it has been unreasonable to conduct the same type of study with traditional experimental test.

Johansson and van Hees (2014) used numerical experiments with FDS 5.5.3 in order to obtain a correlation that could be used to predict gas temperatures in a room adjacent to a room involved in a pre-flashover fire (see Figure 2). It was not considered possible to develop such a correlation with empirical data from traditional experiments due to the lack of resources in terms of laboratory space and time. Instead FDS was used to gather enough empirical data to be able to conduct a multiple regression analysis. The room and opening dimensions, properties of the boundaries and the heat release rate were varied and included as independent variables in a correlation. The process used was similar to what Tilley et al. (2012) used. Johansson and van Hees (2014) considered FDS 5.5.3 to be well validated for the intended purpose of study. However, the correlation derived with the regression analysis was compared with some results from previously published traditional full-scale experiments in order to get an understanding of the external validity. The procedure used to identify the correlation can be termed as a "black box method", i.e. a best fit to data was done without much consideration of the physical meaning of the derived formula. But, Johansson and van Hees (2014) did, as mentioned, compare the formula with some experiments and that is considered curial when using this type of approach.

A similar approach was used in a later study by Johansson et al. (2014b) but with the application to ceiling jets under an unobstructed ceiling with FDS 6.0. A simplified ceiling jet theory, based on a simple energy and mass balance, together with a number of assumptions was used in order to derive an expression for the ceiling jet temperature. This theoretical expression included some unknown constants that could be found with the help of a regression analysis on the data from a numerical experiment. The combination of a simple theoretical model and numerical experiments, with a wellvalidated model, resulted in a final expression that gave a good fit to the FDS simulations and that had a physical basis. The vertical temperature distribution in the ceiling jet was also studied in the paper (see Figure 3) something that is difficult to measure with conventional thermocouples without affecting the gas flow under the ceiling.

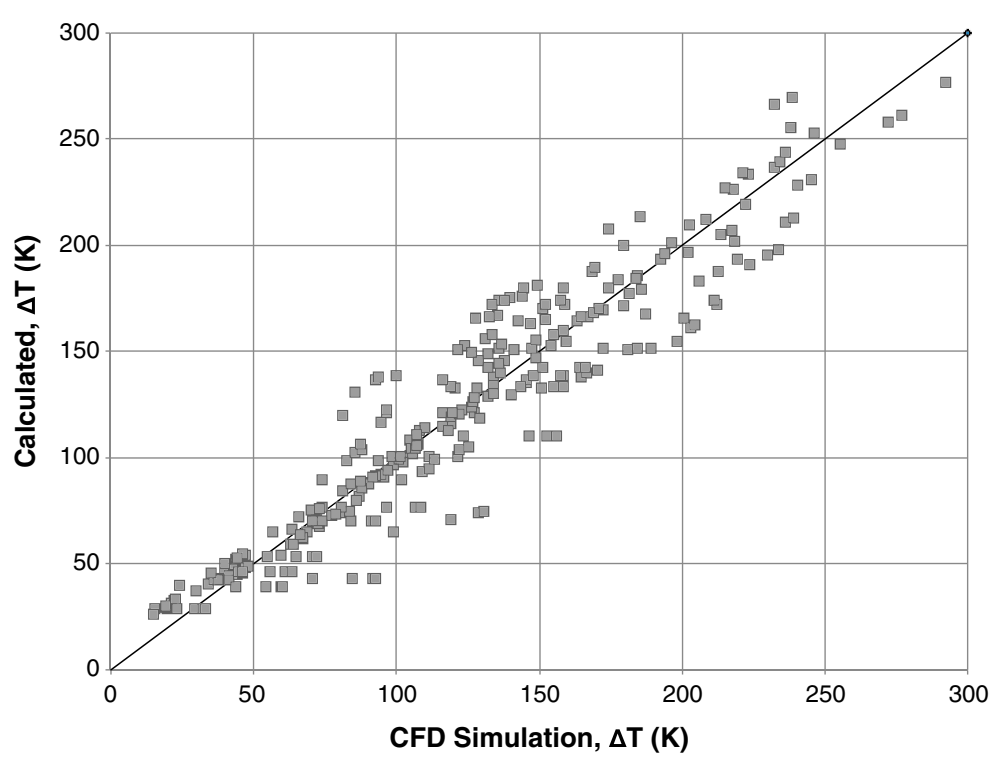

Figure 2 Example of correlation between CFD simulations and an empirical correlation found with a numerical experiment, reproduced from (Johansson and van Hees 2014). 


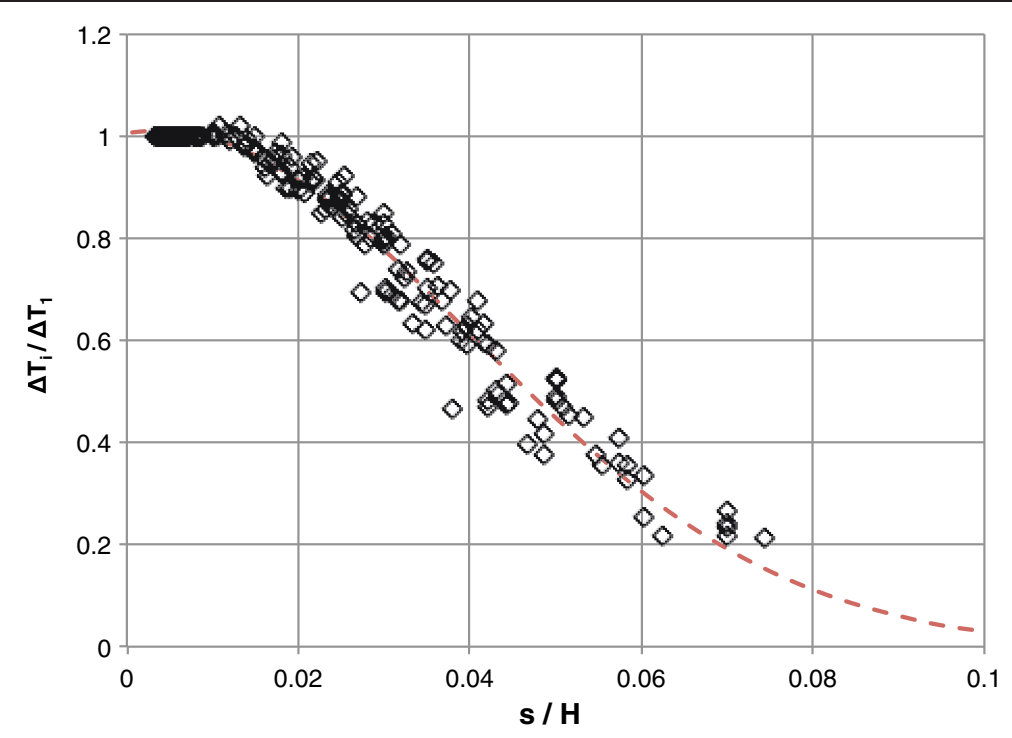

Figure 3 Normalized temperature profile under the ceiling in a numerical experiment conducted with FDS at a distance of $\mathrm{r} / \mathrm{H}=0.9$ from the plume centreline. The data indicates that the cross section temperature in the ceiling jet decreases according to a Gaussian profile (red dotted line). The figure is reproduced from (Johansson et al. 2014b).

\section{Accuracy in numerical experiments}

It is not considered possible to discuss numerical experiments without commenting on factors that might affect the accuracy of the numerical experiment. There are several sources of uncertainty in a numerical experiment. The input parameters used, the competence of the user and the accuracy of the model will all be of importance for the results.

\section{Input parameters}

The input parameters in a fire model are subject to some degree of uncertainty in fire modelling. The uncertainty will be propagated through the fire model, and the quality of such an analysis then depends on the quality of the input data (Lundin 1999). What input parameters that are of importance are dependent on the type of analysis that is conducted. The heat release rate is often regarded as an important parameter in fire safety modelling (Babrauskas and Peacock 1992). In validation exercises of models is the prescribed heat release rate often taken from the experiment, which the comparison is made against (Najafi et al. 2007; van Hees 2013). If the heat release rate is unknown and needs to be modelled it will of course introduce uncertainties that can be large and that needs to be accounted for.

Uncertainties in the input parameters to numerical experiment might be regarded differently. If the numerical experiment is conducted according to Figure 1 will some variables of interest be varied in order to study the correlation to a dependent variable, and the span of variation in the input will determine the area of applicability of the results. There might be input parameters that are kept constant in the numerical experiment but that would have had an influence on the dependent variable. As an example, in the study by $\mathrm{Li}$ and Chow (2003) the ventilation system in a specific tunnel configuration was analysed, thus are the results only valid for that specific tunnel.

\section{User}

In any experiment it is necessary that the researcher is familiar with his or her equipment, how it should be used, how data can be collected and how the data should be interpreted. In a numerical experiment the physics of fires can be interfered with. For instance, different plume models types can be selected in a two-zone model or radiation can be excluded in a CFD model. The user can also introduce error in the numerical experiment due to mistakes. Beard (1997) has elaborated on different types of application error in fire modelling. User effects have also been seen to be of importance in studies with multiple users, such as the PRISME project (Audouin et al. 2011) and in a Swedish verification and validation study (Holmstedt et al. 2008).

\section{Fire model}

In a survey of fire models conducted in 2003 it was concluded that there were roughly 50 different zone models and around 20 field models available for fire modelling (Olenick 2003). No attempt is made in this paper to give an overview of the different models that could be used for numerical experiments. Nor is it within the scope of the paper to describe how well validated or verified the different fire models are. But, it is of course a 
prerequisite for numerical experiments that the fire model used is sufficiently validated for the intended area of study.

An increased accuracy and reduced uncertainty is established in traditional experiments when the random and systematic errors in the experiment are reduced. However, the "true value" of a property in a traditional experiment is not possible to measure exactly and probably not possible to repeat exactly either. In numerical models there is no known "true value" (van Hees 2013). A model can give an estimate of the "true value" and this estimate, can at the best, be considered to be just as good as a measurement in a traditional experiment.

Error and uncertainty cannot be linked together in the same way in models as in traditional experiments. Uncertainty in numerical models arises when there is a potential for a lack of knowledge (AIAA 1998), this means that some deficiencies may or may not exist. Model uncertainties will arise as physical and mathematical assumptions and approximation of the real world are made in different types of models used in fire science (Najafi et al. 2007). On the other hand, errors in numerical models are a recognizable deficiency in any phase or activity of modelling and simulation that is not due to lack of knowledge (AIAA 1998).

There are several different technics that can be used to compare model results with traditional experiments. Descriptive statistics (StatSoft 2013) can be used to retrieve metrics that can be compared, e.g. mean and standard deviation, in two different sets of data. Another method is to visually compare results from modelling and experiments graphically, i.e. to see how well two curves of some measured property corresponds. Such assessments are subjective and qualitatively; furthermore, the determination if there is a match or not is often based on previous experience of what could be regarded as an agreement for a certain parameter. This type of comparisons between experimental results and simulations are presented for a large range of parameters in the FDS validation guide (Floyd et al. 2013b); however, the authors' leaves it to the reader to judge how good the agreement is. The graphical comparisons can also be complemented with a quantitative number of the difference between the two sets of data. This was, for example, done in a comparison of CFAST predictions to real-scale compartment fire tests with natural and forced ventilation (Reneke and Peatross 2001) and it was found that the CFAST model over-predicted upper layer temperatures with less than $50^{\circ} \mathrm{C}$. Another example is the modelling of the Dalmarnock fire tests (Rein et al. 2011) where a posteriori simulations over-predicted the hot layer temperature by $10-50 \%$.

The ASTM guide: "Standard Guide for Evaluating the Predicative Capability of Deterministic Fire Models" (ASTM 2012) describes the steps in the evaluation process of a given model. NRC (Najafi et al. 2007) applied this approach in an evaluation of fire models. In the NRC evaluation the relative difference between model predictions and experimental measurements was studied. This difference, $\varepsilon$, is computed with the following expression:

$$
\varepsilon=\frac{\Delta M-\Delta E}{\Delta E}=\frac{\left(M_{p}-M_{\mathrm{o}}\right)-\left(E_{p}-E_{\mathrm{o}}\right)}{\left(E_{p}-E_{\mathrm{o}}\right)}
$$

Where $\Delta M$ is the difference between the peak value of the model prediction and the baseline and $\Delta E$ is the difference between the peak value of the experimental measurement and the baseline. This relative difference is compared to a combined experimental uncertainty, which included the model input uncertainty and experimental measurement uncertainty.

In the model evaluation performed by NRC five different fire-modelling tools were evaluated for some possible fire scenarios in nuclear facilities. An assessment of the different models accuracy in predicting transport of heat and combustion products in compartments was made in the study. The models capabilities to predict fire growth or fire spread were not studied. A total of 13 output quantities from the models were chosen for the evaluation. The results from the evaluation are presented in the final report (Najafi et al. 2007) in a matrix with a simple colour system, in order to indicate to what degree a certain model predicted a certain output quantity. "Green" indicated that the model predicted a particular parameter with accuracy comparable with the experimental uncertainty and "Yellow" indicated that the predictions were clearly outside the bounds of uncertainty. From the NRC study it is concluded that the studied fire models in general are reasonably accurate in smoke layer temperature and smoke layer depths. For instance, the smoke layer temperature was labelled as "Green" for FDS 4.06, while predictions of smoke concentration was labelled as "Yellow". This mean that FDS 4.06 can be used with confidence to calculate smoke layer temperatures for multi-room compartments, while caution should be exercised when using the model to calculate smoke concentration.

Comparisons at a single time-point can be sufficiently good if peak values or stationary conditions are studied; however, it is not enough if two curves are to be compared. Peacock et al. (1999) have used functional analysis in order to quantify the agreement between different sets of data. Time series curves are treated as multi-dimensional vectors in functional analysis with each point in time defining an additional dimension (ISO 2008). This makes it possible to quantify the length, angle and distance between two curves. This means that, in contrast to the qualitative comparisons, it is possible to compare 
different curves quantitative. Functional analysis has been used previously in evaluation of fire modelling (Peacock et al. 1999) and in evacuation modelling (Galea et al. 2014; Ronchi et al. 2013). Functional analysis has also been included in an example validation procedure in ISO 167301 "Fire safety engineering - Assessment, verification and validation of calculation methods" (ISO 2008).

\section{Advantages and challenges with numerical experiments}

The examples presented in the section above illustrate that numerical models can be used in a manner that can be regarded as an experiment. A system (e.g. car park or compartment) was described in a numerical model in all the examples presented above. Furthermore, the state of that system was changed and the consequence of that change was measured and analysed (as illustrated in Figure 1). These examples shows that numerical experiments can be very useful and that it is a promising method in fire science, but there are of course both advantages and challenges that needs to be highlighted.

\section{Advantages with numerical experiments}

The main advantages with numerical experiments are discussed and motivated in this section.

\section{Resource efficient}

Numerical experiments are much less expensive compared to traditional full-scale experiments. Numerical experiments can be conducted on a personal computer or a computer cluster with free software whereas traditional experiments need to be conducted in a laboratory with a range of measurement equipment that needs calibration. Tens or even hundreds of numerical simulations can be run at the same time, if the computer power is available, while one traditional experimental test usually is carried out at one specific time and after each test the experimental setup needs to be reset. Consequently, numerical experiments create opportunities for more extensive experimental studies and also for a larger research community to contribute to fire science.

Traditional small-scale experiments are more resource efficient compared to full-scale experiments, but it is in most cases not possible to conduct a complete scaling of all dimensionless groups simultaneously. Consequently systematic errors can be introduced when the results are scaled up.

Numerical experiments can be exemplified as more resource efficient than traditional full- and small-scale experiments in two aspects. Firstly, a large number of numerical simulations can be provided to a much lower cost compared to just a single traditional experiment. In Figure 4 it is illustrated how numerical experiments can be used as a complement to traditional experiments. Just

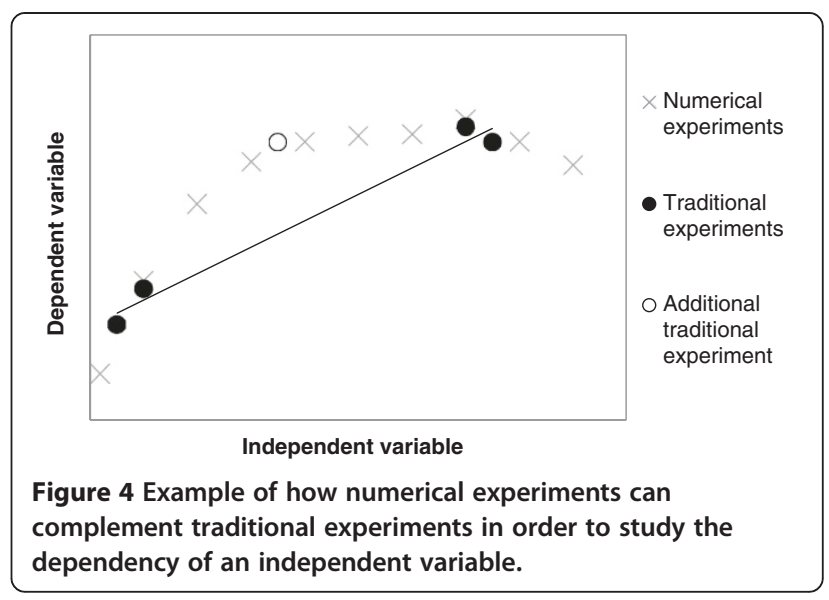

looking at the data from the traditional experiment in Figure 4 indicates that the relationship between the two variables would be linear, but the numerical experiment reveals it to be logarithmical relation. This might not have been possible solely with traditional experiments due to high costs. Instead a single fifth traditional experimentally test should be enough to confirm the relationship found with the numerical experiment. Figure 4 illustrates a regression fit to the data but it can also be a systematic study of different input variables, like the study of roof vents and sprinkler activation by McGrattan et al. (1998), where some simulations were confirmed with traditional experiments.

The second aspect involves the possibility to use numerical experiments to study a large geometry. This was done in the study of the discotheque fire in Gothenburg in 1998 (Ingason et al. 2001). In that study SP conducted small-scale experiments in a laboratory and later used a CFD model to study the problem in the full-scale to confirm the results from the small-scale experiments. It was not possible to conduct a full-scale experiment but the combination of modelling and small-scale experiments gave a credible explanation for how and where the fire started. In a similar way can a numerical experiment be used to extrapolate or confirm some conclusions drawn from a small-scale experimental setup, as illustrated in Figure 5.

It is of course crucial to take care when using a fire model to study a phenomenon in a larger scale or domain as demonstrated in Figure 5 because there is a risk that the study will be outside the limits of the fire model. For instance, the example in Figure 5 could be complemented with a single traditional experiment in the same scale as the numerical experiments, thus confirming the linear trend. In this way are small-scale experiments used together with numerical experiments to form a hypothesis that can be confirmed with a single traditional experiment in full-scale. 


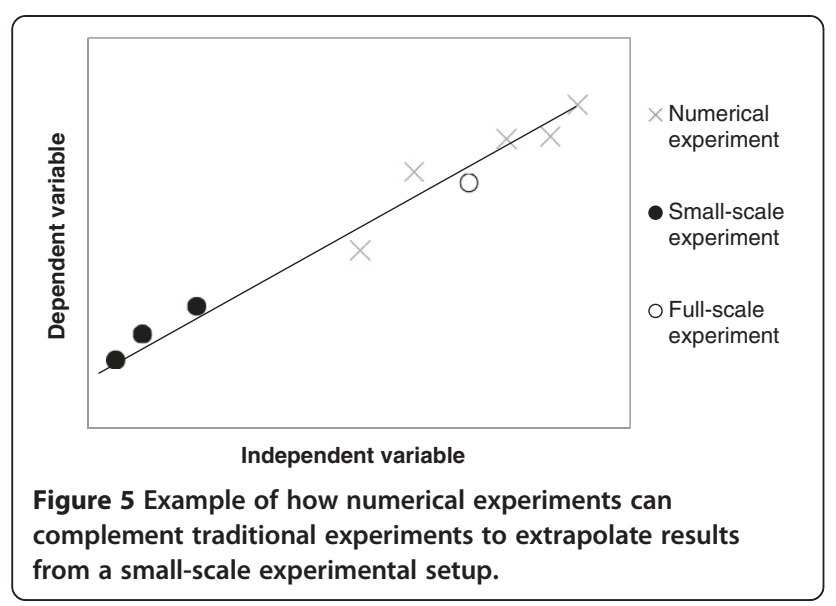

Figure 4 and Figure 5 and the accompanying discussion illustrate that numerical experiments and traditional experiments are complementary and not competitive, and that a combination of these two methods can yield in convincing arguments for some conclusion or hypothesis. A combination traditional experiments and simulations outside the range of validity of the model could also yield in valuable information that can be explored further in future studies.

Another benefit of being resource efficient is that challenging theoretical hypotheses could be tested more easily. Traditional experiments are more expensive than numerical experiments, which mean that the willingness to risk that the result will turn out to be poor or hard to interpret, due to uncertainties, will be less. Numerical experiments might therefore yield in more hypotheses testing in fire science, which in turn can result in more progress in the field.

\section{Level of control of the experiment}

It is probably evident for a researcher if resources are available for traditional experiments, but it might not be as evident if it is possible to control important variables. Several variables of importance might be unknown, which means that there is a risk of systematic errors. Experiments are suitable as a tool for providing information only if the experiment is appropriate and disturbing factors are eliminated. If the experiments are based on wrong or incomplete knowledge it will be problematic, e.g. if knowledge is lacking about how some disturbing factors can be treated or eliminated it could lead to faulty conclusions (Chalmers 1999). An example is ambient conditions like temperature and wind that will have an effect on a traditional experiment. Another example is effects due to how the measurement equipment is placed and calibrated. Such effects were evident in a study Johansson et al. (2014a) were 45 different fire tests analysed in order to study the reproducibility of four different ventilation scenarios in a three-room compartment. The fire tests were well controlled but had been performed during a 6-year period under different ambient conditions. This caused some variation between different tests of the same ventilation scenario as can be seen in Figure 6. Such variation will not be present in a numerical experiment where a higher control of all variables included in the experiment can be kept.

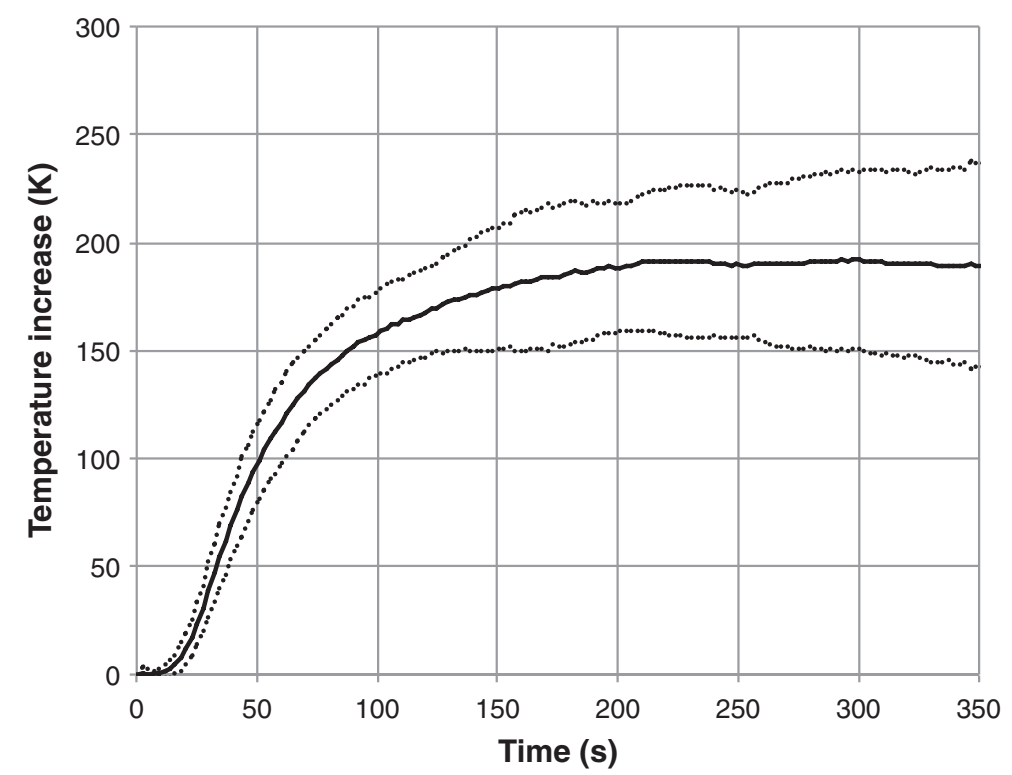

Figure 6 The calculated mean (solid line) and \pm 2 standard deviations (dotted lines) of 15 traditional experiments in a full-scale three-room compartment. The figure is reproduced from Johansson et al. (2014a). 


\section{Measurements}

Measurement equipment is a fundamental part of traditional compartment fire experiments in order to be able to collect data, but the measurement equipment can influence the experiment and the measurements. For example, a thermocouple will give the temperature of the thermocouple and not the gas temperature. Pitts et al. (2003) found that the absolute error in thermocouple measurements due to the radiative environment could be up to $75 \%$ in the lower layer and $7 \%$ in the upper layer. Another example is gas flow measurements with bi-directional probes. If a bi-directional probe is placed in an opening it will influence the gas flow through that opening (Bryant 2005). This influence might be small in some cases but substantial in others e.g. when weak gas flows are studied or when small openings are considered. Even the calorimeter hood and the extraction flow rate can have some influence on the gas flow and local temperature measurements in room fire experiments (Desanghere et al. 2005).

There are far greater possibilities to collect data in a numerical experiment compared to a traditional experiment and without influencing the experiment. If a CFD model like FDS (Floyd et al. 2013a) is used it will be possible to record time dependent information on e.g. temperature or gas concentrations in all cells used in a domain, which could be in millions of different locations. It is impossible to have the same amount of measurements in a traditional experiment. In a numerical experiment it is also possible to record information without adding instrumentation that could influence the experiment, which is still the case in most experiments even though different nonintrusive laser-based measurement technics (Kaldvee et al. 2013) are being introduced.

\section{Challenges with numerical experiments}

The main issues and challenges with numerical experiments are discussed and motivated in the following three sections.

\section{Description of the fire phenomena}

A model is by definition a simplification of the physical reality that includes some approximations. Quintiere (2012) raises several issues with using computer simulation for addressing fire problems. Phenomena like soot formation, fire spread, water droplet breakup and turbulent combustion are things that cannot be addressed adequately with a numerical model (Quintiere 2012). Traditional small- and full-scale experiments capture these types of phenomena. Another issue is fire spread and fire growth. Fire models do provide predictions of different output quantities given a user defined fire but it is difficult to simulate fire spread and fire growth with the current models even with information from experimental measurements (Rein et al. 2011).

This causes issues to the external validity and a distinct limitation of numerical experiments conducted with the fire models currently available. In the evaluation of some selected fire models performed by NRC (Najafi et al. 2007) it was found that gas temperatures, smoke layer heights, room pressure, oxygen concentration and flame heights in compartments could be predicted within or very near the experimental uncertainty of traditional experiments. Therefore, it can be argued that numerical experiments should be limited to these quantities and any other models and quantities that have been as carefully validated.

\section{Experienced user}

A model can be misused and applied outside its limitations. This is problematic because the user has been found to be the most critical link in the chain of simulations (Holmstedt et al. 2008; Keski-Rahkonen et al. 1998). Consequently, the researcher using numerical experiments needs to have an understanding of both the fire phenomena studied and the model used. Many fire models are easy to obtain and easy to use, this means that they can be used in a careless or incorrect manner. This is problematic, because errors due to misuse can be difficult to discover.

The transparency of the numerical experiment may be poor due to the opportunities for the user to change input parameters and use different sub-models. A fundamental part of reporting from experiments is that enough information is available so that the experiment can be replicated with similar results (Andersson 2012). The same should apply for numerical experiments because the experiment must be possible to repeat. This includes the possibility to review how the model has been used and which input parameters and sub-models that have been applied by the researcher. Consequently, it is reasonable to publish relevant parts of the used input file when presenting results from numerical experiments.

\section{Accuracy of fire models}

The evaluation of some selected fire models performed by NRC (Najafi et al. 2007), mentioned above, illustrates that a couple of models can be used to predict a handful of output quantities within the uncertainty bounds of six experimental series. In retrospect of the NRC study it is evident that numerical experiments of compartment fires are currently limited to studying smoke layer temperatures and smoke layer depths. FDS was used in a majority of the examples of conducted numerical experiments given above. However, it is not a necessity to use a CFD model for numerical experiments because more transparent methods like 2-zone models or other 
calculations methods could be appropriate as long as they are sufficiently accurate for the intended purpose.

Even though extensive validation of fire models has been conducted, there is a great deal of work left to do (van Hees 2013). Studies like the one by the NRC (Najafi et al. 2007) reveals that there is a limited area where numerical experiments can be applied. Numerical experiments should not be considered as an alternative to traditional experiments outside this area.

\section{Discussion}

It could be claimed that there is no reasonable argument to replace traditional experiments with numerical experiments if the resources are available and if it is possible to control all variables of importance in the traditional experiments. However, numerical experiments will be much more resource effective if the fire model used is considered to give satisfyingly accurate results for the intended study.

In several of the presented examples the resources for conducting the same amount of full-scale experiments, as were made numerically, were not available and would have been impossible to motivate economically. In for instance the study by Johansson et al. (2014b), it was not an option to reduce the number of experimental tests because a large number of observations were needed in order to perform a satisfying multiple regression analysis. Even performing the same number of observations in small-scale was not considered possible. In the NRC study (Najafi et al. 2007) it was found that FDS could predict compartment fire temperatures and ceiling jet temperatures within the experimental uncertainty. Consequently, the only reasonable experimental method for the study (Johansson et al. 2014b) was a numerical experiment.

The general procedure of conducting the numerical experiment should not be different from that of any other type of experiment. Experimental procedures like for instance described by Andersson (2012) including a planning, data collection and analysis and synthesis phases are of course applicable for numerical experiments. Moreover, the same types of procedures for data analysis, like graphical and statistical methods, can be used. Data from any type of experiment can be imported into a statistical software package in order to use a regression analysis to derive a correlation. Such correlation could be useful but should be handled carefully, because it will have a limited theoretical and physical meaning. This is considered especially important when it comes to numerical experiments because the data comes from a model that contains some simplification or estimation of reality. It is preferable if the results are related to some theory. For example, Hwang and Edwards (2005) found that the results from their numerical experiment could be related to a previously published fire-plume theory, and Johansson et al. (2014b) derived a simple ceiling jet theory and the data from a numerical experiment was used to quantify some constants in order to derive a correlation for the ceiling jet temperature. The numerical experiments in these cases provided numerical results consistent with some theory.

There is no experimental method that can be recommended for use for all types of research tasks in fire science and the experimental methods discussed in this paper all have their strengths and weaknesses. Traditional experiments and numerical experiments are complementary and not competitive and a combination of different types of experimental methods could be necessary to analysis a certain phenomena. Consequently, numerical experiments are considered to play an important roll in fire science in the future. Two examples of how numerical experiments can complement traditional experiments are given in Figure 4 and Figure 5, these are idealised examples but illustrate that a combination of the methods can yield in convincing and resource efficient conclusions.

This paper tries to give directions for the future use of numerical experiments in fire science. The systematic approach and transparency that have been used in the mentioned examples will hopefully serve as an inspiration of how to conduct future numerical experiments in fire science. However, there are several issues that are considered important to study further. This includes the user uncertainty and the accuracy of fire models.

The quality of the numerical experiment will depend on the model used but also on the quality of the experimentalist. Different experimentalists will construct an experiment differently and this is also the case when setting up the experiment in a fire model. It would therefore be appropriate to conduct studies of blind or a prior simulations of predefined scenarios in order to quantify and get an estimate of uncertainty in compartment fire modelling in general and with certain fire models.

In this paper a couple of different validation studies are mentioned, but a lot more validation work has been conducted and more examples are for instance given by van Hees (2013). The examples given in this paper refers primarily to fires in compartments, but there are other areas where numerical experiments could be applied and this could be explored in future research.

More validation work is also needed as current fire models develop and new models and sub-models are presented. There is a tendency to validate the fire model as a whole only, but it is also important to conduct subvalidation, e.g. validation of individual sub-models, because errors in sub-models can be merged in the overall model in a way that cannot be predicted when only the entire model is validated. Sub-model validation is central in the AIAA guide for verification and validation of CFD models (AIAA 1998) and it is something that has been used in the validation process of the CFD model ISIS (Suard et al. 2006). 


\section{Conclusion}

Computer models that can be used to simulate fires and smoke spread have developed rapidly during the last decades. Some of these models have been shown to give predictions of some compartment fire properties within the bounds of measurement uncertainty of performed experiments. Consequently, numerical experiments emerge as a possible complement to traditional compartment fire experiments.

In this paper, numerical experiments are explored as a research method and put into the context of traditional compartment fire experiments. Both pros and contras of numerical experiments compared to traditional compartment fire experiments are presented and summarised in the paper. Numerical experiments are a promising method in fire science research. However, it is currently not considered satisfying to solely use a numerical experiment to find a correlation for a certain compartment fire phenomena without checking the correlation with data from some traditional experiments.

Using the term numerical experiments and recognizing fire models as a tool for experiments in fire science will possibly lead to that established experimental procedures used when planning, performing and analysing traditional experiments also will be applied when performing numerical experiments. The requirements on studies with numerical experiments from the scientific community might also increase if numerical simulations are acknowledged as an experimental method in fire science and this will hopefully result in that the quality of future numerical experiments will be high.

\section{Competing interests}

The author declares that he has no competing interests.

\section{Acknowledgement \\ The financial sponsors: The Swedish Fire Research Board (Brandforsk) and NBSG (the Swedish Nuclear Power Plants Fire Safety Group) are gratefully acknowledged. The author would also like to acknowledge professor Patrick van Hees at the Department of Fire Safety Engineering at Lund University for providing valuable comments on this work.}

Received: 5 May 2014 Accepted: 13 September 2014

Published online: 19 September 2014

\section{References}

AIAA (1998) Guide for the Verification and Validation of Computational Fluid Dynamics Simulations. Reston, VA, USA

Alpert RL (1975) Turbulent Ceiling-Jet induced by Large-Scale Fires. Combust Sci Technol 11:197-213, doi:10.1080/00102207508946699

Andersson O (2012) Experiment! John Wiley \& Sons, doi:10.1002/9781118311059

ASTM (2012) Standard Guide for Evaluating the Predicative Capability of Deterministic Fire Models., doi:10.1520/E1355-12

Audouin L, Chandra L, Consalvi J-L, Gay L, Gorza E, Hohm V, Hostikka S, Ito T, Klein-Hessling W, Lallemand C, Magnusson T, Noterman N, Park JS, Peco J, Rigollet L, Suard S, van Hees P (2011) Quantifying differences between computational results and measurements in the case of a large-scale well-confined fire scenario. Nucl Eng Des 241:18-31, doi:10.1016/j. nucengdes.2010.10.027
Babrauskas V, Peacock R (1992) Heat release rate: the single most important variable in fire hazard. Fire Safety J 18(3):255-272, doi:10.1016/ 0379-7112(92)90019-9

Beard AN (1997) Fire models and design. Fire Safety J 28(2):117-138, doi:10.1016/S0379-7112(96)00082-3

Blurock E, Battin-leclerc F (2013) Modeling Combustion with Detailed Kinetic Mechanisms. In: Battin-Leclerc F, Simmie JM, Blurock E (eds) Cleaner Combustion and Sustainable World. Springer, London, UK, pp 17-57

Bowman KP, Sacks J, Chang YF (1993) Design and Analysis of Numerical Experiments. J Atmos Sci 50:1267-1278, doi:10.1175/1520-0469 (1993) $050<1267:$ DAAONE $>2.0 . C O ; 2$

Bryant RA (2005) Particle Image Velocimetry Measurements of Buoyancy Induced Flow Through a Doorway. National Institute of Technology, Gaithersburg, MD, USA

Chalmers A (1999) What is this thing called science? 3rd edn. Open University Press, Queensland, Australia

Chow WK, Zou GW (2005) Correlation equations on fire-induced air flow rates through doorway derived by large eddy simulation. Build Environ 40:897-906, doi:10.1016/j.buildenv.2004.09.010

Desanghere S, Joyeux D, Coppalle A (2005) Use Of A Numerical Tool To Assess The Impact Of The Means Of Measurement On Fire. Fire Safety Sci 8:1301-1311, doi:10.3801/IAFSS.FSS.8-1301

Floyd J, Forney G, Hostikka S, Korhonen T, McDermott R, McGrattan K, Weinschenk C (2013a) Fire Dynamics Simulator User's Guide. NIST Special Publication 1018, National Institute of Technology, Gaithersburg, MD, USA

Floyd J, Forney G, Hostikka S, Korhonen T, McDermott R, McGrattan K, Weinschenk C (2013b) Fire Dynamics Simulator Technical Reference Guide - Volume 3: Validation. NIST Special Publication 1019, National Institute of Technology, Gaithersburg, MD, USA

Galea ER, Deere S, Brown R, Filippidis L (2014) An Evacuation Validation Data Set for Large Passenger Ships. In: Weidmann U, Kirsch U, Schreckenberg M (eds) Pedestrian and Evacuation Dynamics 2012. Springer International Publishing, doi:10.1007/978-3-319-02447-9

Holmstedt G, Bengtsson S, Blomqvist P, Dittmer T, Hägglund B, Tuovinen H, van Hees P (2008) Quality assurance of accident and injury prevention with fire safety in buildings. Department of Fire Safety Engineering. Lund University, Lund, Sweden

Höst M, Regnell B, Runeson P (2006) Att genomföra examensarbete [Conducting Master Thesis]. Studentlitteratur, Lund, Sweden

Hwang CC, Edwards JC (2005) The critical ventilation velocity in tunnel fires - a computer simulation. Fire Safety J 40(3):213-244, doi:10.1016/j. firesaf.2004.11.001

Ingason H, Wickström U, van Hees P (2001) The Gothenburg Discotheque Fire Investigation. In: The 9th International Conference on Fire Science and engineering, 2nd edn. Interscience Communications, Edinburgh, Scotland, pp 965-976

ISO (2002) Reaction-to-fire tests - Heat release, smoke production and mass loss rate - Part 1: Heat release rate (cone calorimeter method). ISO 5660-1, International Organization for Standardization, Geneva, Switzerland, pp 5660-5661

ISO (2008) Fire safety engineering - Assessment, verification and validation of calculation methods. ISO 16730:2008, International Organization for Standardization, Geneva, Switzerland

Johansson N, van Hees P (2014) A correlation for predicting smoke layer temperature in a room adjacent to a room involved in a pre-flashover fire. Fire Mater 38:182-193, doi:10.1002/fam.2172

Johansson N, Wahlqvist J, van Hees P (2012) Detection of a Typical Arson Fire Scenario - Comparison Between Experiments and Simulations. J Fire Prot Eng 22:23-44, doi:10.1177/1042391511431508

Johansson N, Svensson S, van Hees P (2014a) A Study of Reproducibility of a Full-Scale Multi-Room Compartment Fire Experiment. Fire Technol, doi:10.1007/s10694-014-0408-3

Johansson N, Wahlqvist J, van Hees P (2014b) Numerical experiments in fire science A study of ceiling jets. Fire Mater, Advance online publication 10.1002/fam.2253

Kaldvee B, Wahlqvist J, Jonsson M, Brackmann C, Andersson B, van Hees P, Bood J, Aldén M (2013) Room-Fire Characterization Using Highly Range-Resolved Picosecond Lidar Diagnostics and CFD Simulations. Combust Sci Technol 185:749-765, doi:10.1080/00102202.2012.750310

Kawagoe K (1958) Fire Behavior in Rooms. Building Research Institute, Tokyo, Japan 
Keski-Rahkonen O, Björkman J, Hostikka S, Mangs J, Huhtanen R (1998) Fire Safety. In: Jussi S, Sarkimo M, Merja A, Avall A (eds) Program on the Structural Integrity of Nuclear Power Plants Synthesis of Achievements 1995-1998. VTT, Espo, Finland, pp 281-307

Li JS, Chow W (2003) Numerical studies on performance evaluation of tunnel ventilation safety systems. Tunn Undergr Space Technol 18(5):435-452, doi:10.1016/S0886-7798(03)00023-3

Lundin J (1999) Model Uncertainty in Fire Safety Engineering. Department of Fire Safety Engineering. Lund University, Lund, Sweden

Magnusson SE, Thelandersson S (1970) Temperature-Time Curves of Complete Process of Fire Development - A Theoretical Study of Wood Fuel in Enclosed Spaces. Bulletin of Division of Structural Mechanics and Concrete Construction. Lund Institute of Technology, Lund, Sweden

McGrattan K, Hamins A, Stroup D (1998) Sprinkler, Smoke \& Heat Vent, Draft Curtain Interaction - Large Scale Experiments and Model Development. Retrieved from http://fire.nist.gov/bfrlpubs/fire98/PDF/f98069.pdf

McGrattan K, Hamins A, Forney G (2000) Modeling Of Sprinkler, Vent And Draft Curtain Interaction. Fire Safety Sci 6:505-516, doi:10.3801/IAFSS.FSS.6-505

Najafi D, Jolgar F, Dreisbach J (2007) Verification and Validation of Selected Fire Models for Nuclear Power Plant Applications, Volume 1: Main report. U.S. Nuclear Regulatory Commission, Rockville, MD, USA

Nakaya I, Tanaka T, Yoshida M, Stechler K (1986) Doorway flow induced by a propane fire. Fire Safety J 10(3):185-195, doi:10.1016/0379-7112(86)90015-9

Olenick SM (2003) An Updated International Survey of Computer Models for Fire and Smoke. J Fire Prot Eng 13:87-110, doi:10.1177/1042391503013002001

Peacock RD, Reneke PA, Davis WD, Jones WW et al (1999) Quantifying fire model evaluation using functional analysis. Fire Safety J 33(3):167-184, doi:10.1016/S0379-7112(99)00029-6

Pitts WM, Braun E, Peacock RD, Milter HE, Johnsson EL, Reneke PA, Blevins LG (2003) Temperature Uncertainties for Bare-Bead and Aspirated Thermocouple Measurements in Fire Environments. In: Gritzo LA, Alvares N (eds) Thermal Measurements - The Foundation of Fire Standards. American Society for Testing and Materials International, Dallas, Texas, USA, pp 3-15

Prasad K, Patnaik G, Kailasanath K (2000) Advanced Simulation Tool for Improved Damage Assessment 1) A Multiblock Technique for Simulating Fire and Smoke. US Naval Research Laboratory, Washington DC, USA

Prasad K, Patnaik G, Kailasanath K (2002) A numerical study of water-mist suppression of large scale compartment fires. Fire Safety J 37(6):569-589, doi:10.1016/S0379-7112(02)00004-8

Quintiere JG (1989) Scaling applications in fire research. Fire Safety J 15(1):3-29, doi: 10.1016/0379-7112 (89) 90045-3

Quintiere JG (2006) Fundamentals of Fire Phenomena. John Wiley \& Sons, doi:10.1002/0470091150

Quintiere JG (2012) The Use of Scale Modeling in Fire Safety Design \& Investigation. In: 9th International Conference on Performance Based Codes and Fire Safety Design Methods. Society of Fire Protection Engineering, Hong Kong, China

Rein G, Wolfram J, Torero J (2011) Modelling of the Growth Phase of Dalmarnock Fire Test One. In: 12th International Fire and Materials Conference. Interscience Communication, San Francisco, USA

Reneke P, Peatross M (2001) A comparison of CFAST predictions to USCG real-scale fire tests. Journal of Fire Protection Engineering 11(1):43-68, doi:10.1106/HH4D-0CKM-J53X-FQK1

Robson C (2002) Real World Research, 2nd edn. Blackwell, Oxford, UK

Ronchi E, Reneke P, Peacock RD (2013) A Method for the Analysis of Behavioural Uncertainty in Evacuation Modelling. Fire Technol 2, doi:10.1007/s10694-013-0352-7

StatSoft I (2013) Electronic Statistics Textbook. StatSoft, Tulsa, USA, Retrieved from http://www.statsoft.com/textbook

Suard S, Audouin L, Babik F (2006) Verification and validation of the ISIS CFD code for fire simulation. Workshop on Assessment of Calculation Methods in Fire Safety Engineering, Southwest Research Institute, San Antonio, Texas, USA, Retrieved from http://www.latp.univ-mrs.fr/ latche/Confs/iso_06.pdf
Tilley N, Rauwoens P, Merci B (2011) Verification of the accuracy of CFD simulations in small-scale tunnel and atrium fire configurations. Fire Safety J 46:186-193, doi:10.1016/J.Firesaf.2011.01.007

Tilley N, Deckers X, Merci B (2012) CFD study of relation between ventilation velocity and smoke backlayering distance in large closed car parks. Fire Safety J 48:11-20, doi:10.1016/J.Firesaf.2011.12.005

Van Hees P (2013) Validation and Verification of Fire Models for Fire Safety Engineering. Procedia Eng 62:154-168, doi:10.1016/j.proeng.2013.08.052

doi:10.1186/s40038-014-0002-2

Cite this article as: Johansson: Numerical experiments and compartment fires. Fire Science Reviews 2014 3:2.

\section{Submit your manuscript to a SpringerOpen ${ }^{\circ}$ journal and benefit from:}

- Convenient online submission

- Rigorous peer review

- Immediate publication on acceptance

- Open access: articles freely available online

- High visibility within the field

- Retaining the copyright to your article

Submit your next manuscript at $>$ springeropen.com 